\title{
A computational approach based on ordinary state-based peridynamics with new transition bond for dynamic fracture analysis
}

\author{
Michiya Imachi ${ }^{a}$, Satoyuki Tanaka ${ }^{a}$, Tinh Quoc Bui ${ }^{b}$, \\ Selda Oterkus ${ }^{\mathrm{c}}$, Erkan Oterkus ${ }^{\mathrm{c}}$ \\ ${ }^{a}$ Graduate School of Engineering, Hiroshima University, Japan, \\ e-mail:d174722@hiroshima-u.ac.jp,satoyuki@hiroshima-u.ac.jp \\ ${ }^{b}$ Department of Civil and Environmental Engineering, \\ Tokyo Institute of Technology, Japan, e-mail: bui.t.aa@m.titech.ac.jp \\ ${ }^{c}$ Department of Naval Architecture, Ocean and Marine Engineering, \\ Unversity of Strathclyde, United Kingdom, \\ e-mail:selda.oterkus@strath.ac.uk,erkan.oterkus@strath.ac.uk
}

\begin{abstract}
The recently developed ordinary state-based peridynamics (OSPD) is further enhanced to study elastodynamic propagating crack based on the dynamic stress intensity factors (DSIFs). The displacement discontinuity such as a crack surface is represented by a bond-failure. Variations of the mixed-mode DSIFs with time are evaluated by the interaction integral method for the dynamic crack propagation. In terms of OSPD fracture modeling, numerical oscillation of DSIFs becomes a critical issue during the evolution of a crack. To overcome this numerical oscillation problem, we introduce a new model of bond-failure, the transition bond. The enhanced OSPD approach using the new transition bond model offers accurate and acceptable results, suppressing the numerical oscillation of responses and reflecting an effective approach. The effects of different types of transition bond are numerically analyzed. Accuracy of the DSIFs is examined employing the various damping parameters and effectiveness of the new PD fracture model is verified. The Kalthoff-Winkler impact test is considered for evaluating the mixed-mode DSIFs and the crack paths.
\end{abstract}

Keywords: Peridynamics; Dynamic Fracture; Dynamic Stress Intensity Factors; Crack Propagation 


\section{Introduction}

Evaluation of dynamic crack propagation in solids and structures is a long-standing issue in the physics and engineering mechanics research fields. Dynamic fracture and crack propagation can critically damage the structure. Therefore, quantitative evaluation of the dynamic fracture phenomena is essential for structural safety and reliability assessment. Dynamic fracture problems have been investigated by a number of researchers, and the achievements are summarized in Refs. [1,2]. Dynamic fracture includes intrinsically difficult issues, e.g., transient, fast moving boundary, dynamic loading, crack branching, and rate- and material-dependent problems. Further investigations are required to clarify such complicated physical phenomena.

Recently, computational fracture mechanics has become a powerful tool for the evaluation of fracture mechanics behavior. The finite element method (FEM) [3], eXtended FEM (X-FEM) [4], and meshfree method [5,6] have been used to evaluate fracture mechanics parameters. Aoki et al. $[7,8]$ and Kishimoto et al. [9] used mode-I dynamic stress intensity factor (DSIF) to evaluate dynamic crack propagation using singular elements. Dynamic crack propagation problems have also been analyzed using X-FEM under mixedmode loading conditions [10-13].

The aforementioned numerical methods were formulated based on classical continuum mechanics theory. Although the methods can be used to accurately analyze several fracture problems, there are certain drawbacks in treating crack initiation, branching, and fragmentation problems. Peridynamics (PD), which is a non-classical continuum mechanics approach proposed by Silling [14], has potential in dealing with fracture mechanics problems. In analyzing cracked solids and structures, particles are scattered throughout the body. An interaction relationship is established between each particle within a horizon. The interaction is called a bond in the PD framework. A force state is defined for each bond to represent the bond forces between material points in the PD theory. Fracture can be modeled by breaking the bond, i.e., bond failure. Currently, the PD theory can be categorized into bond-based PD, ordinary state-based PD (OSPD), and non-ordinary statebased PD based on the force state description $[15,16]$. Dual-horizion PD was also proposed to analyze different spatial resolution problems effectively for varying horizon size $[17,18]$.

The prototype microelastic brittle (PMB) model [15] has been employed for the fracture criterion in the PD theory and brittle materials have been 
analyzed [19-22]. Alternatively, the DSIFs can be utilized in the PD theory. The fracture mechanics parameters are well established based on classical fracture mechanics theory and are suitable for discussing dynamic fracture behaviors. By introducing DSIF evaluation in the PD theory, crack branching phenomena can be analyzed. In our previous studies [23,24], the dynamic J-integral and the mixed-mode DSIFs of a stationary crack under dynamic loading were accurately evaluated using a two-dimensional (2D) OSPD [25]. A moving least squares approximation (MLSA) [26] and meshfree discretization technique, i.e., the diffraction method [27], were introduced to accurately evaluate the physical values around the crack tip.

In the present study, DSIF evaluation was extended for dynamic crack propagation problem. However, numerical oscillations were found during crack propagation when evaluating the DSIFs. The oscillations become serious when crack propagation direction is evaluated by maximum circumferential stress criterion. Therefore, a new PD fracture model reducing numerical oscillations was proposed for elastodynamic crack propagation problems. The problem was carefully examined and a damping effect was introduced in the bond failure model. Mode-I and mixed-mode fracture problems were investigated and the effectiveness of the present PD fracture model and the DSIF evaluation technique is discussed. Although some methodologies have been proposed for evaluating fracture parameters in PD theories [28,29], no studies have addressed the numerical oscillations for dynamic fracture problems to get accurate DSIFs.

The paper is organized as follows. The 2D OSPD is briefly introduced in Section 2. The mixed-mode DSIF evaluation technique and fracture criteria for dynamically propagating cracks are presented in Section 3. In Section 4, a mode-I problem is analyzed. A damping effect is introduced into the bond failure model and the effectiveness is examined. A mixed-mode problem is then examined to verify the effectiveness of the proposed PD fracture model. Conclusions are presented in Section 5. 


\section{Peridynamic theory}

\subsection{Basic theory}

The basic formulation of PD theory can be derived from the equation of motion [16]. The equation of motion at position vector $\boldsymbol{x}$ in a finite body can be written as

$$
\rho \ddot{\boldsymbol{u}}(\boldsymbol{x}, t)=\int_{\mathcal{H}_{x}}\left[\underline{\boldsymbol{T}}(\boldsymbol{x}, t)\left\langle\boldsymbol{x}^{\prime}-\boldsymbol{x}\right\rangle-\underline{\boldsymbol{T}}\left(\boldsymbol{x}^{\prime}, t\right)\left\langle\boldsymbol{x}-\boldsymbol{x}^{\prime}\right\rangle\right] \mathrm{d} V_{\boldsymbol{x}^{\prime}}+\boldsymbol{b}(\boldsymbol{x}, t),
$$

where $\rho$ is the mass density, $\ddot{\boldsymbol{u}}(\boldsymbol{x}, t)$ is the acceleration vector, and $\boldsymbol{b}(\boldsymbol{x}, t)$ is the external force vector per unit volume. In PD modeling, a finite body is modeled by scattered particles. The particles located at $\boldsymbol{x}$ have a volume $\mathrm{d} V_{\boldsymbol{x}} . \underline{\boldsymbol{T}}(\boldsymbol{x}, t)\left\langle\boldsymbol{x}^{\prime}-\boldsymbol{x}\right\rangle(=\underline{\boldsymbol{T}})$ and $\underline{\boldsymbol{T}}\left(\boldsymbol{x}^{\prime}, t\right)\left\langle\boldsymbol{x}-\boldsymbol{x}^{\prime}\right\rangle\left(=\underline{\boldsymbol{T}}^{\prime}\right)$ are the force density vectors of particles located at $\boldsymbol{x}$ and $\boldsymbol{x}^{\prime}$, respectively, which are interacting with each other. A particle interacts with other particles within an influence radius, given by the horizon $\delta . \mathcal{H}_{x}$ is the neighborhood of the particle located at $\boldsymbol{x}$.

In OSPD modeling, the force state can be written as

$$
\underline{T}=\underline{t m},
$$

where $\underline{\boldsymbol{m}}$ is a unit direction vector, $\underline{\boldsymbol{m}}=(\boldsymbol{\xi}+\boldsymbol{\eta}) /|\boldsymbol{\xi}+\boldsymbol{\eta}| . \boldsymbol{\xi}\left(=\boldsymbol{x}-\boldsymbol{x}^{\prime}\right)$ and $\boldsymbol{\eta}\left(=\boldsymbol{u}-\boldsymbol{u}^{\prime}\right)$ are the relative position and displacement vectors, respectively. $\underline{t}$ stands for the magnitude of the force density and its definition for the plane stress is given [25], by

$$
\begin{gathered}
\underline{t}=\frac{2(2 \nu-1)}{\nu-1}\left(\kappa^{\prime} \theta-\frac{\alpha}{3}\left(\underline{\omega e^{\mathrm{d}}}\right) \bullet|\underline{\boldsymbol{\xi}}|\right) \frac{\underline{\omega}|\underline{\boldsymbol{\xi}}|}{(\underline{\omega}|\underline{\boldsymbol{\xi}}|) \bullet|\underline{\boldsymbol{\xi}}|}+\alpha \underline{\omega e}^{\mathrm{d}}, \\
\theta=\frac{2(2 \nu-1)}{\nu-1} \frac{(\underline{\omega}|\underline{\boldsymbol{\xi}}|) \bullet \underline{e}}{(\underline{\omega}|\underline{\boldsymbol{\xi}}|) \bullet|\underline{\boldsymbol{\xi}}|}, \\
\alpha=\frac{8 G}{(\underline{\omega}|\underline{\boldsymbol{\xi}}|) \bullet|\underline{\boldsymbol{\xi}}|}, \\
\kappa^{\prime}=K+\frac{G(\nu+1)^{2}}{9(2 \nu-1)^{2}},
\end{gathered}
$$

and for plane strain condition, by

$$
\underline{t}=2\left(\kappa^{\prime} \theta-\frac{\alpha}{3} \frac{\omega e^{\mathrm{d}}}{\bullet}|\underline{\boldsymbol{\xi}}|\right) \frac{\underline{\omega}|\underline{\boldsymbol{\xi}}|}{(\underline{\omega}|\underline{\boldsymbol{\xi}}|) \bullet|\underline{\boldsymbol{\xi}}|}+\alpha \underline{\omega e^{\mathrm{d}}},
$$




$$
\begin{gathered}
\theta=2 \frac{(\underline{\omega}|\underline{\boldsymbol{\xi}}|) \bullet \underline{e}}{(\underline{\omega}|\underline{\boldsymbol{\xi}}|) \bullet|\underline{\boldsymbol{\xi}}|}, \\
\alpha=\frac{8 G}{(\underline{\omega}|\underline{\boldsymbol{\xi}}|) \bullet|\underline{\boldsymbol{\xi}}|}, \\
\kappa^{\prime}=K+\frac{G}{9},
\end{gathered}
$$

where $\nu, G$ and $K$ are Poisson's ratio, the elastic shear and bulk moduli, respectively. $\underline{\omega}$ is an arbitrary influence function which depends on the distance between particles. $\underline{e}^{\mathrm{d}}$ and $\theta$ are deviatoric part of the extension state and the volume dilatation. 


\section{Evaluation of DSIFs and fracture modeling}

According to linear fracture mechanics theory, materials fail if the energy of crack growth is larger than the resistance of material. SIFs can express the state of the crack tip and it is well known that there is a certain relationship between the SIFs and energy release rate. In the present study, critical stress intensity factor $K_{\text {Ic }}$ is adopted for the fracture toughness based on the classical fracture mechanics theory.

\subsection{Interaction integral for dynamic crack propagation}

Mixed-mode DSIFs are evaluated for dynamic propagating cracks. The interaction integral method was introduced into the OSPD for the stationary crack problems under the dynamic loading condition in [24]. The interaction integral method for dynamic propagating cracks proposed by Réthoré et al. [10] is employed. The velocity field near the crack tip is required to evaluate the interaction integral for a dynamic propagating crack. The interaction integral $I$ is given by

$$
\begin{aligned}
& I=\int_{\Omega} q_{1, j} {\left[\left(\sigma_{i j}^{\mathrm{aux}} u_{i, k}^{\mathrm{act}}+\sigma_{i j}^{\mathrm{act}} u_{i, k}^{\mathrm{aux}}\right)-\left(\sigma_{m l}^{\mathrm{aux}} u_{m, l}^{\mathrm{act}}-\rho \dot{u}_{l}^{\mathrm{act}} \dot{u}_{l}^{\mathrm{aux}}\right) \delta_{k j}\right] } \\
&+q_{1}\left[\left(\sigma_{i j, j}^{\mathrm{aux}} u_{i, k}^{\mathrm{act}}+\sigma_{i j, j}^{\mathrm{act}} u_{i, k}^{\mathrm{aux}}\right)+\rho\left(\dot{u}_{i}^{\mathrm{aux}} \dot{u}_{i, k}^{\mathrm{act}}+\dot{u}_{i}^{\mathrm{act}} \dot{u}_{i, k}^{\mathrm{aux}}\right)\right] \mathrm{d} \Omega,
\end{aligned}
$$

where the superscripts "act" and "aux" indicate the actual and auxiliary field variables, respectively. $\sigma_{i j}, u_{i}$ and $\dot{u}_{i}$ are the component of stress, displacement and velocity. $\Omega$ is the integration domain and $\delta_{k j}$ is the Kronecker delta. The spatial derivative of the variables is evaluated using MLSA. $q_{1}$ is a weight function for evaluation of the domain integral, as

$$
q_{1}= \begin{cases}1 & r_{q}<r_{q 1} \\ 1-\left(r_{q}-r_{q 1}\right) /\left(r_{q 2}-r_{q 1}\right) & r_{q 1}<r_{q}<r_{q 2} \\ 0 & \text { otherwise }\end{cases}
$$

where $r_{q}$ is the distance from the crack tip and $r_{q 1}$ and $r_{q 2}$ are arbitrary values that satisfy $r_{q 1}<r_{q 2}$.

Additionally, the interaction integral $I$ can be represented by DSIFs with the actual and auxiliary fields using the relationship between the energy release rate and SIF as follows

$$
I=\frac{2}{E^{*}}\left(A_{\mathrm{I}}(\dot{a}) K_{\mathrm{I}}^{\mathrm{act}} K_{\mathrm{I}}^{\mathrm{aux}}+A_{\mathrm{II}}(\dot{a}) K_{\mathrm{II}}^{\mathrm{act}} K_{\mathrm{II}}^{\mathrm{aux}}\right),
$$


where $A_{\mathrm{I}}(\dot{a})$ and $A_{\mathrm{II}}(\dot{a})$ are the universal functions that depend on the crack speed $\dot{a}$. The universal functions are defined by Freund [1] as

$$
\begin{aligned}
& A_{\mathrm{I}}(\dot{a})=\frac{4 \alpha_{d}\left(1-\alpha_{s}^{2}\right)}{(\kappa+1) D}, \\
& A_{\mathrm{II}}(\dot{a})=\frac{4 \alpha_{s}\left(1-\alpha_{s}^{2}\right)}{(\kappa+1) D},
\end{aligned}
$$

where $\kappa=(3-\nu) /(1+\nu), E^{*}=E$ and $\kappa=3-4 \nu, E^{*}=E /\left(1-\nu^{2}\right)$ are defined for the plane stress and plane strain conditions, respectively. $\alpha_{d}, \alpha_{s}$, and $D$ are determined as

$$
\begin{gathered}
\alpha_{d}=\sqrt{1-\frac{\dot{a}^{2}}{c_{d}^{2}}}, \\
\alpha_{s}=\sqrt{1-\frac{\dot{a}^{2}}{c_{s}^{2}}}, \\
D=4 \alpha_{d} \alpha_{s}-\left(1+\alpha_{s}^{2}\right)^{2} .
\end{gathered}
$$

$c_{d}$ and $c_{s}$ are the longitudinal and transverse wave speeds, respectively.

\subsection{Failure criterion and propagation direction}

The crack propagation direction is evaluated by the maximum circumferential stress criterion [30], which states the crack propagates toward the direction on which the circumferential stress is maximum. Consequently, the DSIF of mode-I and mode-II cracks become maximum and zero, respectively. The direction $\theta_{c}$ of the crack propagation is represented by DSIFs $K_{\mathrm{I}}$ and $K_{\text {II }}$ as

$$
\theta_{c}=2 \arctan \left[\frac{1}{4}\left\{\frac{K_{\mathrm{I}}}{K_{\mathrm{II}}}-\operatorname{sgn}\left(K_{\mathrm{II}}\right)\left(\frac{K_{\mathrm{I}}^{2}}{K_{\mathrm{II}}^{2}}+8\right)^{\frac{1}{2}}\right\}\right] .
$$

In case of the single-mode problem, if DSIF $K_{\mathrm{I}}$ is greater than the critical SIF $K_{\text {Ic }}$, the crack propagates straight. For a mixed-mode crack problem, the equivalent SIF $K_{\theta_{c}}$ is compared with $K_{\text {Ic }}$. If $K_{\theta_{c}} \geq K_{\text {Ic }}$, the crack propagates toward the direction $\theta_{c}$. $K_{\theta_{c}}$ is given by

$$
K_{\theta_{c}}=\cos ^{3}\left(\frac{\theta_{c}}{2}\right) K_{\mathrm{I}}-\frac{3}{2} \cos \left(\frac{\theta_{c}}{2}\right) \sin \left(\theta_{c}\right) K_{\mathrm{II}} .
$$




\section{Discretization}

\subsection{Discretization of peridynamic formula}

The equation of motion in Eq.(1) is discretized, for the $i$-th particle as

$$
\rho_{(i)} \ddot{\boldsymbol{u}}_{(i)}=\sum_{j}^{N}\left(\underline{\boldsymbol{T}}_{(i)(j)}-\underline{\boldsymbol{T}}_{(j)(i)}\right) A_{(j)}+\boldsymbol{b}_{(i)},
$$

where the subscripts $(i)$ and $(j)$ indicate variables of $i$ - and $j$-th particle, and $\underline{\boldsymbol{T}}_{(i)(j)}$ and $\underline{\boldsymbol{T}}_{(j)(i)}$ are the force density of $i$ - and $j$-th particles interact each other. $N$ is the number of particles in neighborhood. The central difference scheme is used for the time integration and the equation of motion can be written as

$$
\boldsymbol{u}_{(i)}^{n+1}=\frac{\Delta t^{2}}{\rho_{(i)}}\left[\sum_{j}^{N}\left(\underline{\boldsymbol{T}}_{(i)(j)}^{n}-\underline{\boldsymbol{T}}_{(j)(i)}^{n}\right) A_{(j)}+\boldsymbol{b}_{(i)}^{n}\right]+2 \boldsymbol{u}_{(i)}^{n}-\boldsymbol{u}_{(i)}^{n-1},
$$

where $\Delta t$ is the time interval and the superscripts $n-1, n$ and $n+1$ indicate variables at $(n-1)$-, $(n)$ - and $(n+1)$-th step. The magnitude of the force state between $i$ - and $j$-th particles for plane stress in discretized form can be written as

$$
\begin{array}{r}
\underline{t}_{(i)(j)}=\frac{2(2 \nu-1)}{\nu-1}\left(\kappa_{(i)}^{\prime} \theta_{(i)}-\frac{\alpha_{(i)}}{3} \sum_{j}^{N} \underline{\omega}_{(i)(j)} \underline{e}_{(i)(j)}^{\mathrm{d}}\left|\underline{\boldsymbol{\xi}}_{(i)(j)}\right| A_{(j)}\right) \\
\times \frac{\underline{\omega}_{(i)(j)}\left|\underline{\boldsymbol{\xi}}_{(i)(j)}\right|}{\sum_{j}^{N} \underline{\omega}_{(i)(j)}\left|\underline{\boldsymbol{\xi}}_{(i)(j)}\right|\left|\underline{\boldsymbol{\xi}}_{(i)(j)}\right| A_{(j)}}+\alpha_{(i)} \underline{\omega}_{(i)(j)} \underline{\boldsymbol{e}}_{(i)(j)}^{\mathrm{d}},
\end{array}
$$

with

$$
\theta_{(i)}=\frac{2(2 \nu-1)}{\nu-1} \frac{\sum_{j}^{N} \underline{\omega}_{(i)(j)}\left|\underline{\boldsymbol{\xi}}_{(i)(j)}\right| \underline{\boldsymbol{e}}_{(i)(j)}^{d} A_{(j)}}{\sum_{j}^{N} \underline{\omega}_{(i)(j)}\left|\underline{\boldsymbol{\xi}}_{(i)(j)}\right|\left|\underline{\boldsymbol{\xi}}_{(i)(j)}\right| A_{(j)}}
$$


where $\underline{\omega}_{(i)(j)}$ is the influence function between $i$ - and $j$-th particle. The fracture modeling is controlled by introducing an on/off bond condition in the standard PD theory. If the bond of $i$ - and $j$-th particle is broken, the influence function becomes zero $\left(\omega_{(i)(j)}=0\right)$. This bond condition can be written by scalar value function $f_{b}$ as follows:

$$
f_{b}=\left\{\begin{array}{ll}
1 & \text { unbroken bond } \\
0 & \text { broken bond }
\end{array} .\right.
$$

Consequently, the influence function considering a bond breakage is defined $\underline{\hat{\omega}}_{(i)(j)}=f_{b} \cdot \underline{\omega}_{(i)(j)}$, then Eqs. (23) and (24) can be rewritten by

$$
\begin{array}{r}
\underline{t}_{(i)(j)}=\frac{2(2 \nu-1)}{\nu-1}\left(\kappa_{(i)}^{\prime} \theta_{(i)}-\frac{\alpha_{(i)}}{3} \sum_{j}^{N} \underline{\hat{\omega}}_{(i)(j)} \underline{e}_{(i)(j)}^{\mathrm{d}}\left|\underline{\boldsymbol{\xi}}_{(i)(j)}\right| A_{(j)}\right) \\
\times \frac{\underline{\hat{\omega}}_{(i)(j)}\left|\underline{\boldsymbol{\xi}}_{(i)(j)}\right|}{\sum_{j}^{N} \underline{\hat{\omega}}_{(i)(j)}\left|\underline{\boldsymbol{\xi}}_{(i)(j)}\right|\left|\underline{\boldsymbol{\xi}}_{(i)(j)}\right| A_{(j)}}+\alpha_{(i)} \underline{\hat{\omega}}_{(i)(j)} \underline{e}_{(i)(j)}^{\mathrm{d}},
\end{array}
$$

with

$$
\theta_{(i)}=\frac{2(2 \nu-1)}{\nu-1} \frac{\sum_{j}^{N} \underline{\hat{\omega}}_{(i)(j)} \underline{\boldsymbol{\xi}}_{(i)(j)} \mid \underline{\boldsymbol{e}}_{(i)(j)}^{d} A_{(j)}}{\sum_{j}^{N} \underline{\hat{\omega}}_{(i)(j)}\left|\underline{\boldsymbol{\xi}}_{(i)(j)}\right|\left|\underline{\boldsymbol{\xi}}_{(i)(j)}\right| A_{(j)}} .
$$

The bond conditions $f_{b}=1$ and $f_{b}=0$ are respectively called "connected bond" and "disconnected bond", in this paper. Fig.1(b) and (c) represent an important aspect in crack propagation modeling with the PD approach. Originally, a connected bond becomes a disconnected bond when the line segment of the bond crosses the crack segment. This issue is schematically sketched in Fig.1(b). Once the crack starts propagating, the connected bond also becomes a disconnected bond as shown in Fig.1(c). 


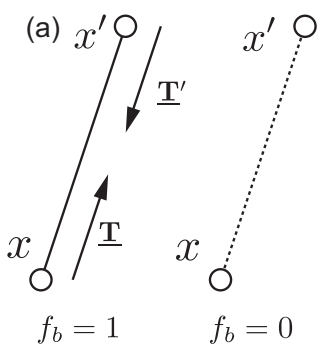

(b)

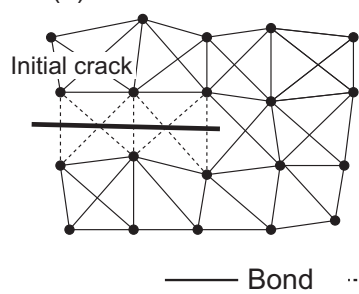

(c) Crack propagation $K_{\theta_{c}} \geq K_{I c}$

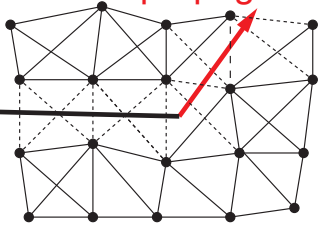

Disconnected bond

Figure 1: Schematic of crack modeling: (a) bond force model with a scalar function $f_{b}$, (b) the initial crack, and (c) a propagating crack.

\subsection{Interaction integral}

The interaction integral $I$ in Eq.(11) is discretized as

$$
\begin{aligned}
I=\sum_{n}^{N P} q_{(n) 1, j}[ & \left(\sigma_{(n) i j}^{\mathrm{aux}} u_{(n) i, k}^{\mathrm{act}}+\sigma_{(n) i j}^{\mathrm{act}} u_{(n) i, k}^{\mathrm{aux}}\right) \\
- & \left.\left.\sigma_{(n) m l}^{\mathrm{aux}} u_{(n) m, l}^{\mathrm{act}}-\rho_{(n)} \dot{u}_{(n) l}^{\mathrm{act}} \dot{u}_{(n) l}^{\mathrm{aux}}\right) \delta_{k j}\right] \\
& +q_{(n) 1}\left[\left(\sigma_{(n) i j, j}^{\mathrm{aux}} u_{(n) i, k}^{\mathrm{act}}+\sigma_{(n) i j, j}^{\mathrm{act}} u_{(n) i, k}^{\mathrm{aax}}\right)\right. \\
& \left.+\rho_{(n)}\left(\dot{u}_{(n) i}^{\mathrm{aux}} \dot{u}_{(n) i, k}^{\mathrm{act}}+\dot{u}_{(n) i}^{\mathrm{act}} \dot{u}_{(n) i, k}^{\mathrm{aux}}\right)\right] A_{(n)} .
\end{aligned}
$$

$N P$ is number of particles for computing the interaction integral $I$. Practically, the interaction integral is used for separation to classical fracture modes. The actual DSIFs of mode-I and -II can be computed by assuming the auxiliary field as the pure mode-I $\left(K_{\mathrm{I}}^{\text {aux }}=1, K_{\mathrm{II}}^{\text {aux }}=0\right)$ and -II $\left(K_{\mathrm{I}}^{\text {aux }}=0, K_{\mathrm{II}}^{\mathrm{aux}}=1\right)$ condition, as follows

$$
\begin{aligned}
K_{\mathrm{I}}^{\mathrm{act}} & =\frac{E^{*}}{2 A_{\mathrm{I}}(\dot{a})} I_{\mathrm{I}}, \\
K_{\mathrm{II}}^{\mathrm{act}} & =\frac{E^{*}}{2 A_{\mathrm{II}}(\dot{a})} I_{\mathrm{II}},
\end{aligned}
$$

where, $I_{\mathrm{I}}$ and $I_{\mathrm{II}}$ are the interaction integral assuming the auxiliary field as the pure mode-I and -II and their discretization form can be written as 


$$
\begin{aligned}
& I_{\mathrm{I}}=\sum_{n}^{N P} q_{(n) 1, j}\left[\left(\sigma_{\mathrm{I}(n) i j}^{\mathrm{aux}} u_{(n) i, k}^{\mathrm{act}}+\sigma_{(n) i j}^{\mathrm{act}} u_{\mathrm{I}(n) i, k}^{\mathrm{aux}}\right)\right. \\
& \left.-\left(\sigma_{\mathrm{I}(n) m l}^{\mathrm{aux}} u_{(n) m, l}^{\mathrm{act}}-\rho_{(n)} \dot{u}_{(n) l}^{\mathrm{act}} \dot{u}_{\mathrm{I}(n) l}^{\mathrm{aux}}\right) \delta_{k j}\right] \\
& +q_{(n) 1}\left[\left(\sigma_{\mathrm{I}(n) i j, j}^{\mathrm{aux}} u_{(n) i, k}^{\mathrm{act}}+\sigma_{(n) i j, j}^{\mathrm{act}} u_{\mathrm{I}(n) i, k}^{\mathrm{aux}}\right)\right. \\
& \left.+\rho_{(n)}\left(\dot{u}_{\mathrm{I}(n) i}^{\mathrm{aux}} \dot{u}_{(n) i, k}^{\mathrm{act}}+\dot{u}_{(n) i}^{\mathrm{act}} \dot{u}_{\mathrm{I}(n) i, k}^{\mathrm{aux}}\right)\right] A_{(n)}, \\
& I_{\mathrm{II}}=\sum_{n}^{N P} q_{(n) 1, j}\left[\left(\sigma_{\mathrm{II}(n) i j}^{\mathrm{aux}} u_{(n) i, k}^{\mathrm{act}}+\sigma_{(n) i j}^{\mathrm{act}} u_{\mathrm{II}(n) i, k}^{\mathrm{aux}}\right)\right. \\
& \left.-\left(\sigma_{\mathrm{II}(n) m l}^{\mathrm{aux}} u_{(n) m, l}^{\mathrm{act}}-\rho_{(n)} \dot{u}_{(n) l}^{\mathrm{act}} \dot{u}_{\mathrm{II}(n) l}^{\mathrm{aux}}\right) \delta_{k j}\right] \\
& +q_{(n) 1}\left[\left(\sigma_{\mathrm{II}(n) i j, j}^{\mathrm{aux}} u_{(n) i, k}^{\mathrm{act}}+\sigma_{(n) i j, j}^{\mathrm{act}} u_{\mathrm{II}(n) i, k}^{\mathrm{aux}}\right)\right. \\
& \left.+\rho_{(n)}\left(\dot{u}_{\mathrm{II}(n) i}^{\mathrm{aux}} \dot{u}_{(n) i, k}^{\mathrm{act}}+\dot{u}_{(n) i}^{\mathrm{act}} \dot{u}_{\mathrm{II}(n) i, k}^{\mathrm{aux}}\right)\right] A_{(n)},
\end{aligned}
$$

where variable that has the superscript "aux" and the subscript "I" and "II" indicates variable of auxiliary field for pure mode-I and -II. Stress is evaluated by collapsed stress tensor, defined by

$$
\boldsymbol{\sigma}_{(n)}^{\text {act }}=\sum_{k}^{N P} \underline{\boldsymbol{T}}_{(n)(k)} \otimes \underline{\boldsymbol{\xi}}_{(n)(k)} A_{(k)} .
$$

The derivative of the displacement and the velocity are evaluated by

$$
\begin{aligned}
& u_{(n) i, k}^{\text {act }}=\phi_{(n) i, k} u_{(n) i}^{\text {act }}, \\
& \dot{u}_{(n) i, k}^{\text {act }}=\phi_{(n) i, k} \dot{u}_{(n) i}^{\text {act }},
\end{aligned}
$$

where $\phi_{(n)}$ is the MLS shape function of $n$-th particle.

\subsection{Moving least squares approximation} by

In MLSA [26], the approximated function $g^{h}(\boldsymbol{x})$ located at $\boldsymbol{x}$ is defined

$$
g^{h}(\boldsymbol{x})=\boldsymbol{p}^{T}(\boldsymbol{x}) \boldsymbol{a}(\boldsymbol{x}),
$$


where $\boldsymbol{p}^{T}(\boldsymbol{x})$ is a basis function and a quadratic basis $\boldsymbol{p}^{T}(\boldsymbol{x})=\left[\begin{array}{ll}1 & x_{1} x_{2} x_{1}^{2} x_{1} x_{2} x_{2}^{2}\end{array}\right]$ is used. $\boldsymbol{a}(\boldsymbol{x})$ is the coefficient so as to minimize the weighted $L_{2}$-norm $Q$, as

$$
\begin{aligned}
Q & =\sum_{i}^{m} w\left(\boldsymbol{x}-\boldsymbol{x}_{i}\right)\left[\boldsymbol{p}^{T}\left(\boldsymbol{x}_{i}\right) \boldsymbol{a}(\boldsymbol{x})-g\left(\boldsymbol{x}_{i}\right)\right]^{2} \\
& =(\boldsymbol{P} \boldsymbol{a}-\boldsymbol{g})^{T} \boldsymbol{W}(\boldsymbol{x})(\boldsymbol{P a}-\boldsymbol{g})
\end{aligned}
$$

where $m$ is the number of particles for approximation. Therefore, the approximated function $g^{h}(\boldsymbol{x})$ can be written by

$$
\begin{aligned}
g^{h}(\boldsymbol{x}) & =\boldsymbol{p}(\boldsymbol{x})^{T} \boldsymbol{C}^{-1}(\boldsymbol{x}) \boldsymbol{D}(\boldsymbol{x}) \boldsymbol{g} \\
& =\boldsymbol{\phi}(\boldsymbol{x}) \boldsymbol{g}
\end{aligned}
$$

where

$$
\begin{gathered}
\boldsymbol{W}(\mathbf{x})=\operatorname{diag}\left[w\left(\boldsymbol{x}-\boldsymbol{x}_{1}\right) \cdots w\left(\boldsymbol{x}-\boldsymbol{x}_{n}\right)\right], \\
\boldsymbol{P}=\left[\begin{array}{cccc}
p_{1}\left(\boldsymbol{x}_{1}\right) & p_{2}\left(\boldsymbol{x}_{1}\right) & \cdots & p_{m}\left(\boldsymbol{x}_{n}\right) \\
p_{1}\left(\boldsymbol{x}_{2}\right) & p_{2}\left(\boldsymbol{x}_{2}\right) & \cdots & p_{m}\left(\boldsymbol{x}_{n}\right) \\
\vdots & \vdots & \ddots & \vdots \\
p_{1}\left(\boldsymbol{x}_{n}\right) & p_{2}\left(\boldsymbol{x}_{n}\right) & \cdots & p_{m}\left(\boldsymbol{x}_{n}\right)
\end{array}\right], \\
\boldsymbol{C}(\boldsymbol{x})=\boldsymbol{W}(\boldsymbol{x}) \boldsymbol{P} \boldsymbol{P}^{T}, \\
\boldsymbol{D}(\boldsymbol{x})=\boldsymbol{W}(\boldsymbol{x}) \boldsymbol{P}, \\
w\left(\boldsymbol{x}-\boldsymbol{x}_{i}\right)=\left\{\begin{array}{cc}
1-\frac{3}{2} s^{2}+\frac{3}{4} s^{3} & (0 \leq s \leq 1) \\
\frac{1}{4}(2-s)^{3} & (1 \leq s \leq 2), \\
0 & (2 \leq s) \\
s=\left|\boldsymbol{x}-\boldsymbol{x}_{i}\right| / \delta .
\end{array}\right.
\end{gathered}
$$

The diffraction method [27] is introduced to describe the near crack-tip. 


\section{Representative numerical examples and discussion}

\subsection{A semi-infinite plate with a crack subjected to tensile loads}

The dynamic propagating crack model is discussed by considering a semiinfinite plate as depicted in Fig.2. The semi-infinite plate is subjected to a tensile load, and this is a mode-I crack problem. The dynamic loading $\sigma_{0}$ is applied on both sides of the surface, and symmetric boundary conditions are adopted. The plate is $40 \mathrm{~mm}$ high and $52 \mathrm{~mm}$ wide and the crack length is $a=12 \mathrm{~mm}$. The Young's modulus is $E=75.629 \mathrm{GPa}$, the Poisson's ratio is $\nu=0.286$, and the mass density is $\rho=2,456 \mathrm{~kg} / \mathrm{m}^{3}$. The particle distance $d l=0.2 \mathrm{~mm}$ and Horizon $\delta=4 d l$ are used. The time interval $\Delta t=2.5 \times 10^{-2} \mu \mathrm{s}$ is chosen for the stable computation. In Eq.(12), $r_{q 1}=2.0 \mathrm{~mm}$ and $r_{q 2}=4.0 \mathrm{~mm}$ are chosen for computing DSIFs. This is known as the major moving crack problem and the analytical solution is defined by Freund [1] as

$$
K_{\mathrm{I}}=2 \sigma_{0} k(v) \frac{\sqrt{c_{d} t(1-2 \nu) / \pi}}{1-\nu} \quad(t>\bar{t}),
$$

where $\bar{t}=H / c_{d}$ and half the plate height $H=20 \mathrm{~mm}$ is used. $k(v)$ is approximated by

$$
k(v) \approx\left(1-v / c_{R}\right) / \sqrt{1-\nu / c_{d}},
$$

where $c_{d}$ and $c_{R}$ are the longitudinal and Rayleigh wave speeds, respectively. The mode-I DSIF is normalized by $\sigma_{0} \sqrt{\pi a}$ as

$$
\bar{K}_{\mathrm{I}}=K_{\mathrm{I}} / \sigma_{0} \sqrt{\pi a} .
$$

In this example, the crack propagation criterion is used as simple condition instead of Eq.(20) for the purpose of comparison of the computed DSIF with the analytical solution. For the crack propagation, the bond failure modeling in Eq.(25) and Fig.1 is used. The crack remains stationary until the critical time $t_{c r}=4.4 \mu \mathrm{s}$, then the crack propagates with a crack velocity $\dot{a}=1,000 \mathrm{~m} / \mathrm{s}$ toward the crack propagation direction $\theta_{c}=0$. The crack propagation criterion can be written as

$$
\dot{a}=\left\{\begin{array}{cc}
0 & t \leq t_{c r} \\
1,000 & t_{c r}<t
\end{array} .\right.
$$


The normalized DSIFs are compared with the analytical solution in Eq.(45) and the reference solution evaluated by Nishioka and Atluri [31]. The results are shown in Fig.3. For the stationary crack, the result is in good agreement with analytical and reference solutions. After the crack propagation, numerical oscillations are found, although inclination of the DSIF agreed well. The crack initiation and propagation direction are evaluated by DSIFs with the maximum circumferential stress theory. The crack propagation direction is evaluated by the ratio of DSIFs of mode-I and mode-II in Eq.(19). The oscillation is significant. After careful examination, the spurious oscillation occurs because of sudden bond release. To overcome this issue, a damping effect model is introduced to suppress the numerical oscillations.

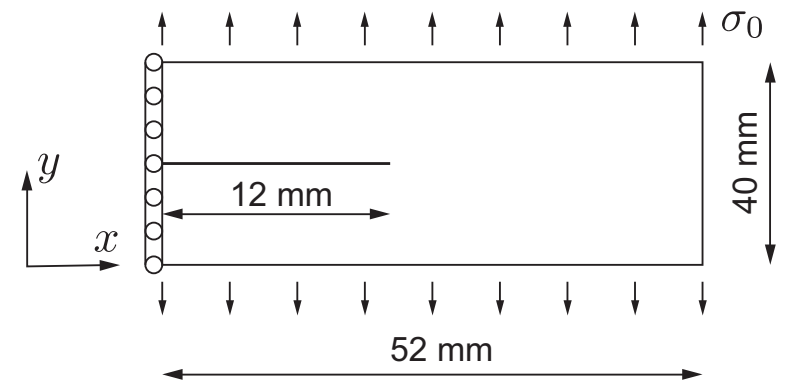

Figure 2: Schematic of the semi-infinite plate.

\subsection{New PD fracture model}

The numerical response of the DSIFs indicates the significance of numerical oscillations when the bond is suddenly released. We introduce a new bond condition called a "transition bond" to suppress the numerical oscillations. The scalar function $f_{b}$ is modified to take a value from 0 to 1 . The function depended on the time $t-t_{c r}$ and the crack velocity $\dot{a} . t-t_{c r}$ is the elapsed time when the bond is broken. The scalar function $f_{b}$ is redefined as

$$
f_{b}(t, \dot{a})=\left\{\begin{array}{ll}
1 & \text { for connected bond } \\
0<f_{b}<1 & \text { for transition bond } \\
0 & \text { for disconnected bond }
\end{array} .\right.
$$

When a crack segment crosses the line segment of a bond, the connected bond becomes a transition bond. An updated crack modeling schematic is shown in Fig.4. 


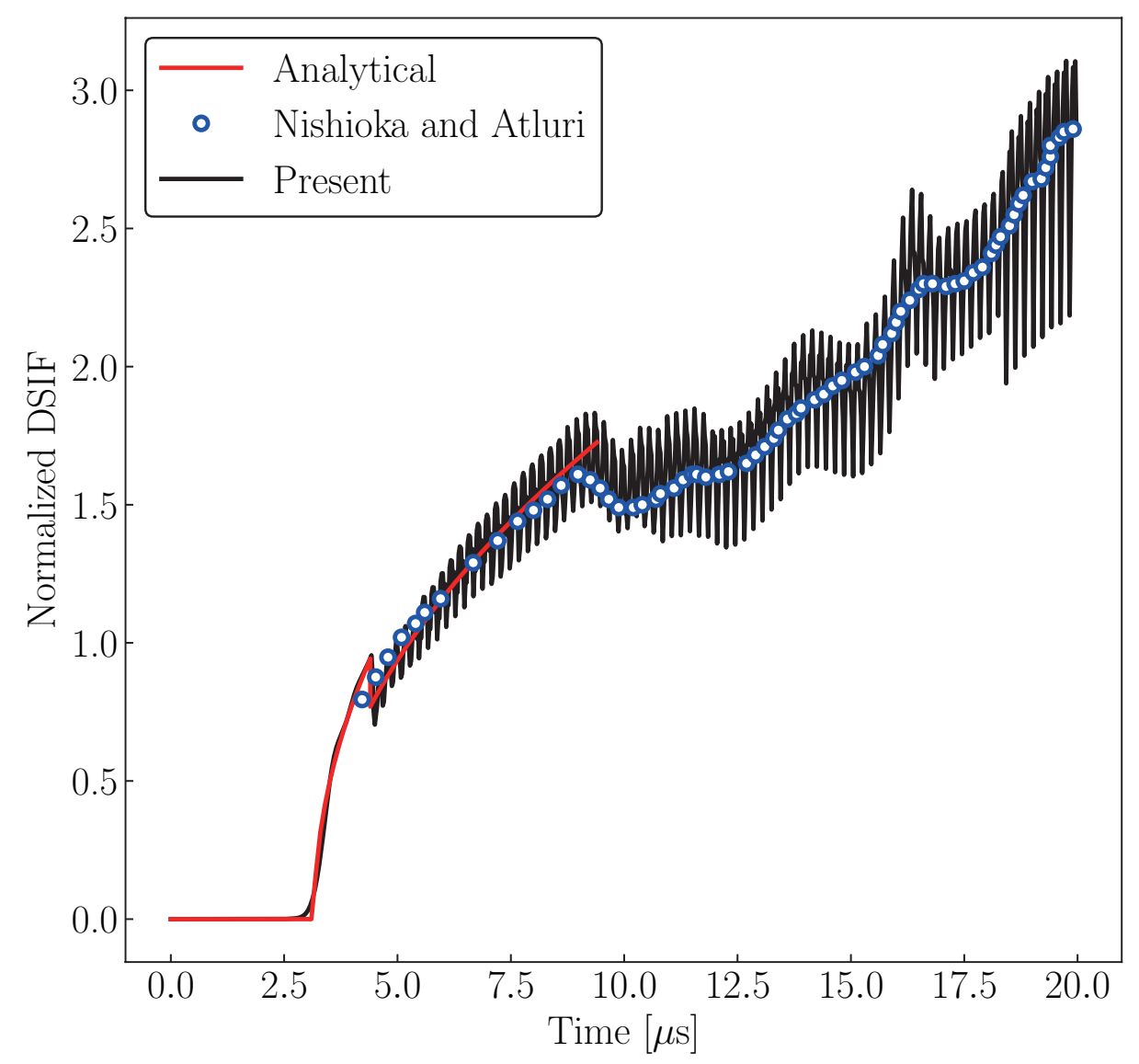

Figure 3: Comparison of normalized DSIFs. 
The transition time is defined as the time at which the crack passes the damping length $\Delta a$ as shown in Fig.5. It takes $f_{b}$ from 1 to $0 . f_{b}$ is 1 just before the crack growth is initiated as the crack growth length $d a=0$ at critical time $t=t_{c r}$. Then, when the crack growth length $d a=\dot{a}\left(t-t_{c r}\right)$ is equal to the damping length $\Delta a, f_{b}$ becomes 0 . The longer the transition time, the smoother the bond failure. We discuss the effect of several types of transition bonds in the next section.

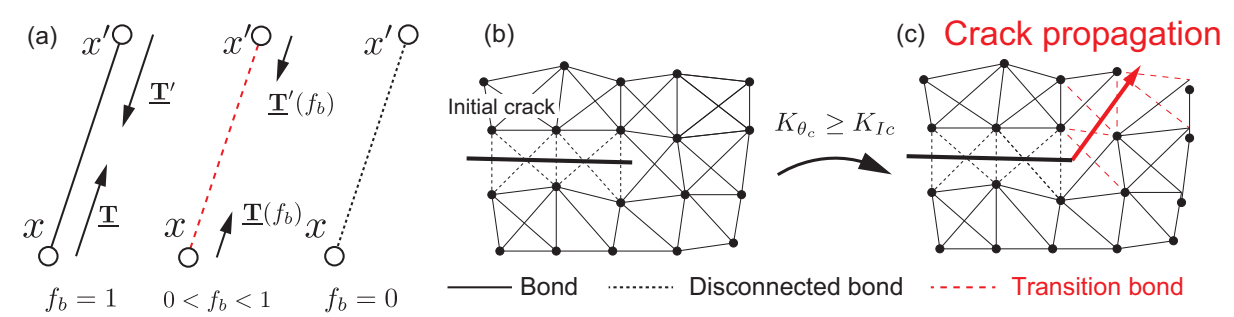

Figure 4: Schematic of crack modeling including the damping model: (a) a bond force modeled with a scalar function $f_{b}$, (b) the initial crack, and (c) a propagating crack.

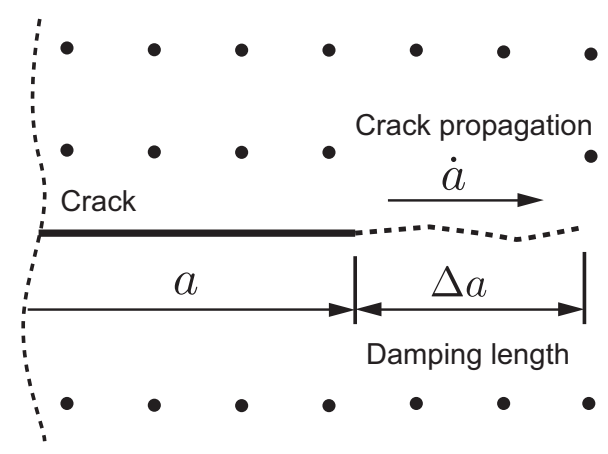

Figure 5: Schematic of crack propagation for defining the damping length.

\subsection{A semi-infinite plate with a crack subjected to tensile loads with the} transition bond

To suppress the numerical oscillations, the transition bond is introduced as the damping model. A basic scalar function is chosen as follows

$$
f_{b}=(1-d a / \Delta a)^{n}
$$


where $\Delta a$ is the damping length and $n$ is the damping coefficient. $f_{b}$ is simple function and it only depends on $\Delta a$ and $n$. The damping length $\Delta a$ controls a damping time. The damping coefficient $n$ controls value of a bond force. If $n=1$ is chosen, a bond force is decreasing linearly. Different damping lengths and damping coefficients are considered to examine the effect of the transition bond model.

Damping lengths $\Delta a=\delta, \delta / 2$, and $\delta / 4$ (at a damping coefficient $n=1$ ) are used to determine the effect of the damping length. The shape of the scalar function $f_{b}$ is shown in Fig.6(a). The normalized DSIFs are shown in Fig.6(b). The oscillations are suppressed well compared with those of suddenly release model. The difference in DSIF values are further investigated at the moment of crack propagation (area A) and after crack propagation (area B) in Fig.6(b). A close-up view of areas A and B are shown in Fig.6(c) and (d), respectively. There are difference in DSIF values among the transition models in Fig.6(c). The crack propagation is delayed when a larger damping length was used. The crack propagation is similar to that of the suddenly release model when a small damping length is used.

Damping coefficients $n=1,2$, and 4 (at a damping length $\Delta a=\delta / 2$ ) is used to determine the effect of the damping coefficient. The shape of $f_{b}$ is shown in Fig.7(a). The results are shown in Fig.7(b)-(d). As expected, the numerical oscillations with the transition bond are suppressed well compared with those that resulted from the suddenly release model in Fig.7(b). There are differences among the three transition models at the moment of crack propagation, as shown in Fig.7(c). When the damping coefficient $n$ is set large, value of $f_{b}$ of left hand side becomes small, as shown in Fig.7(a). Therefore, crack propagation delay is improved. After the crack propagation, there are small differences among the transition bond models, as shown in Fig.7(d). Considering the above results, damping length $\Delta a=\delta / 2$ and damping coefficient $n=4$ are used in the following numerical examples.

The velocity fields computed by the suddenly release and the transition bond models at $t=20.0 \mu \mathrm{s}$ are shown in Fig.8(a) and (b), respectively. Numerical oscillations are found near the crack surface in Fig.8(a). However, numerical oscillations are suppressed with the transition bond model, as shown in Fig.8(b). The interaction integral $I$ are evaluated by the velocity field near the crack tip. The oscillations induce deterioration in DSIFs when the interaction integral are computed.

Moreover, effectiveness of the transition bond is examined using some parameters, for instance, time interval $\Delta t$, horizon $\delta$ and particle distance 
$d l$. Analysis conditions are same as above example except survey parameter for following examples. First, $\Delta t=1.0 \times 10^{-2}, 2.5 \times 10^{-2}$ and $5.0 \times 10^{-2}$ $\mu s$ are used for examining the influence of time interval on numerical oscillation. The results using the suddenly released model and the transition bond for different time interval $\Delta t$ are shown in Fig.9(a) and (b). The different horizon $\delta=3.5 \mathrm{dl}$, $4.0 \mathrm{dl}$ and $4.5 \mathrm{dl}$ are also analyzed. The results of DSIFs between the suddenly released model and the transition bond model are shown in Fig.10(a) and (b). The result of DSIFs for different particle distances $d l=0.5,0.2$ and $0.1 \mathrm{~mm}$ are shown in Fig.11(a) and (b) as well. Numerical oscillation occurred in all cases used the suddenly released model. As expected, numerical oscillation is suppressed well using the transition bond model for different parameters.

Additionally, the accuracy of the DSIFs in an irregular particle arrangement is examined. The irregularity of the particle arrangement is defined by

$$
\boldsymbol{x}_{\mathrm{irr}}=\boldsymbol{x}+d l \cdot r_{c} \cdot \alpha_{\mathrm{irr}},
$$

where $\boldsymbol{x}$ and $\boldsymbol{x}_{\text {irr }}$ are the position vectors for the regular and irregular arrangements, respectively. $d l$ is the particle distance, $r_{c}$ is a randomization factor, and an irregularity factor $\alpha_{\text {irr }}=0.3$ is chosen. Comparison between the analytical solution, reference solution, and the present model are shown in Fig.12. The DSIFs of the regular and irregular arrangements are in good agreement with the analytical and reference solutions.

\subsection{Investigation of load-displacement curve}

The load-displacement curve is examined to further investigate effectiveness of the transition bond model. The analysis model is shown in Fig.13 and tensile speed is applied at point A and B. The dimension and material properties are same as Section 5.1 and time step $\Delta t=2.5 \times 10^{-2} \mu \mathrm{s}$, horizon $\delta=4.0 d l$, particle distance $d l=0.2 \mathrm{~mm}$ are chosen. The tensile speed is increasing gradually until $v_{y}=10 \mathrm{~m} / \mathrm{s}$ for quasi-static loading. The displacement of the loading point becomes $u_{y}=0.1 \mathrm{~mm}$, then the crack propagates with crack speed $\dot{a}=0.01 c_{s} \mathrm{~m} / \mathrm{s}$ for the crack propagation criterion. The load-displacement curve using the suddenly released model and the transition bond model are shown in Fig.14. In the suddenly released model, the reaction force oscillates and the amplitude increases as the time increases. 

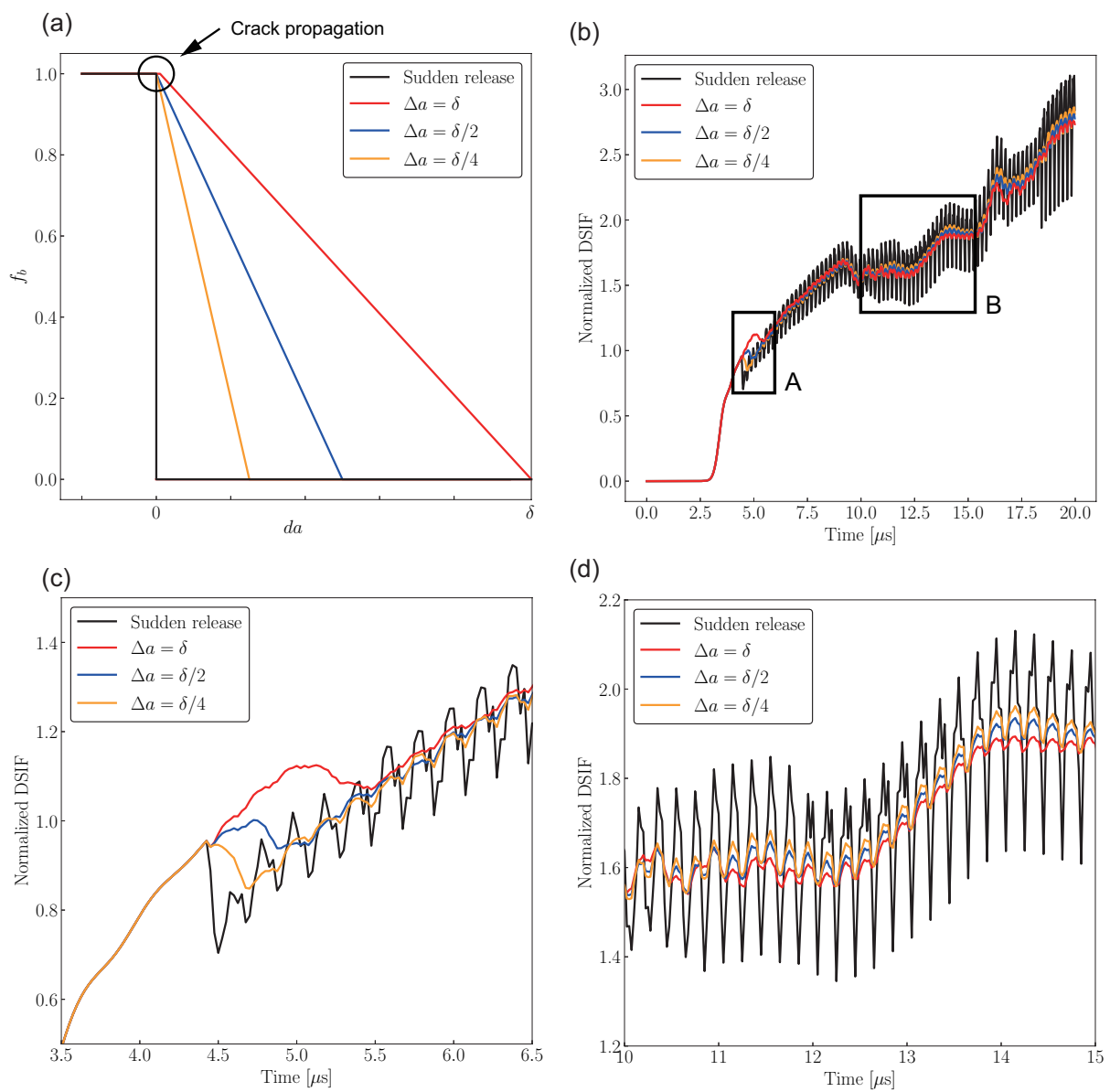

Figure 6: Comparison of normalized DSIFs at several damping lengths $\Delta a$ : (a) shape of $f_{b},(\mathrm{~b})$ overall view, (c) close-up view of area A, and (d) close-up view of area B. 


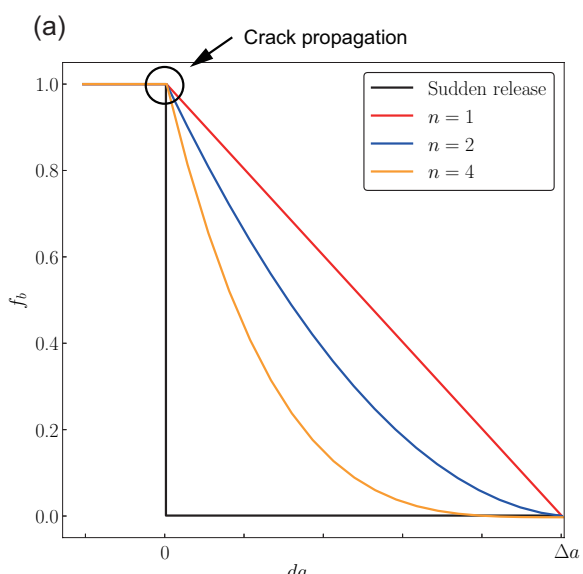

(b)

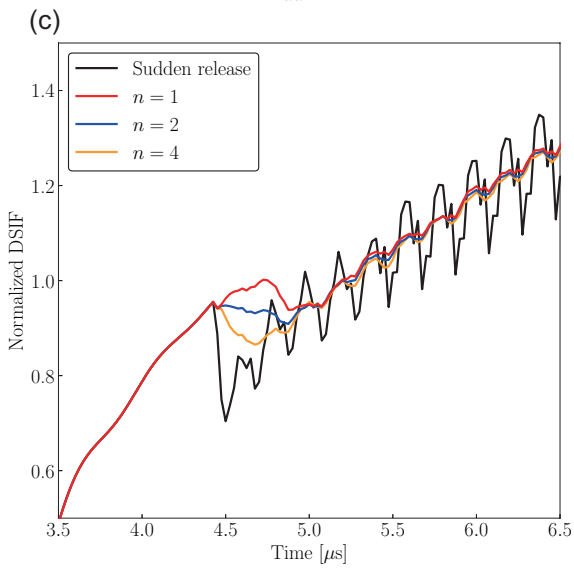

(d)
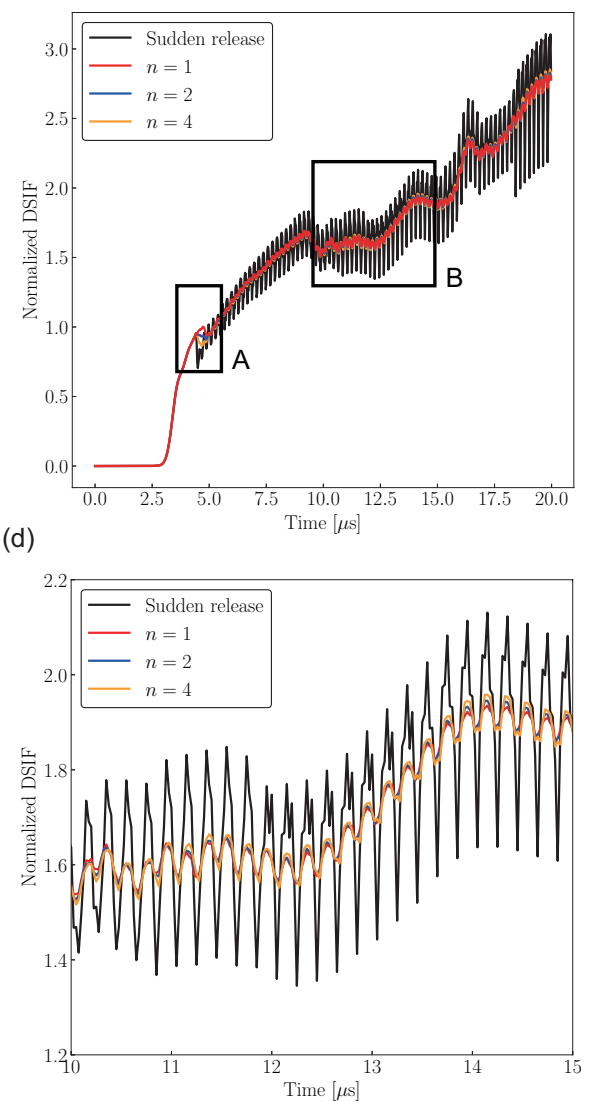

Figure 7: Comparison of normalized DSIFs at several damping coefficients $n$ in Eq.48: (a) shape of $f_{b},(\mathrm{~b})$ overall view, (c) close-up view of area A, and (d) close-up view of area B. 
(a)

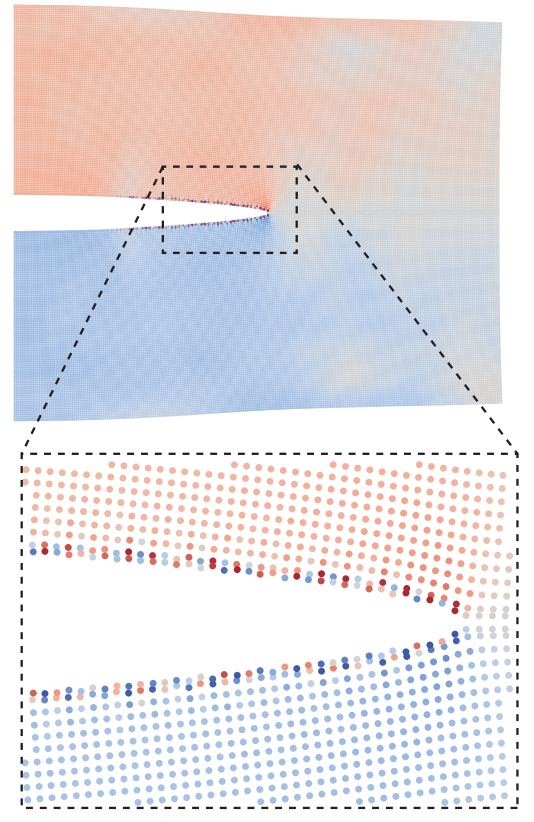

(b)

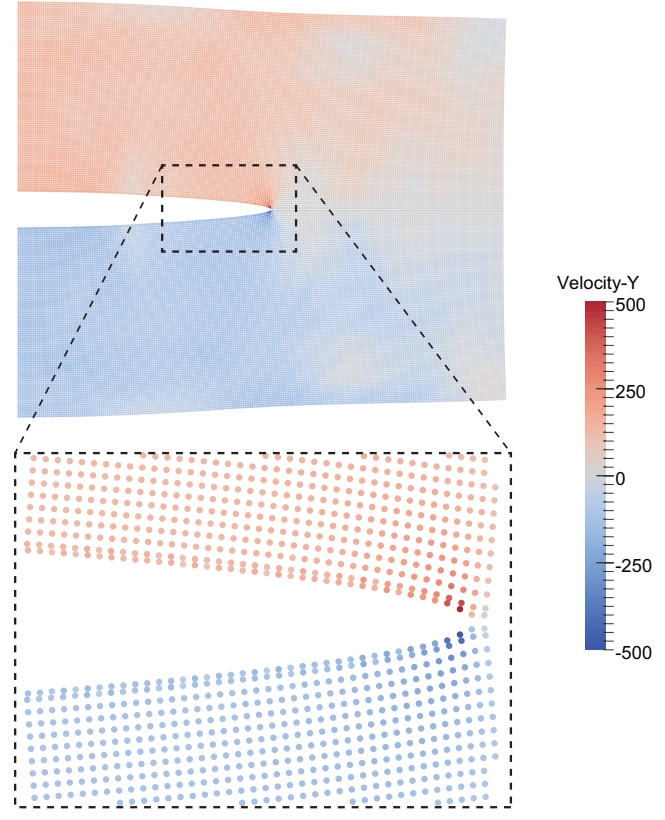

Figure 8: Comparison of velocity fields: (a) regular particle arrangement without the transition bond model and (b) regular particle arrangement with the transition bond model.

(a)

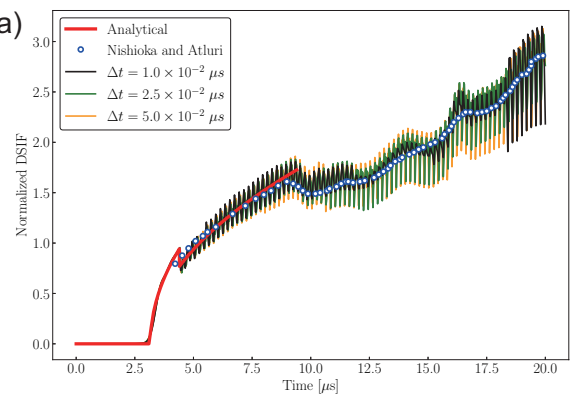

(b)

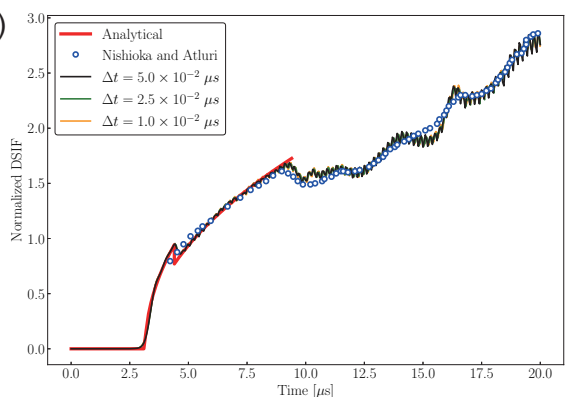

Figure 9: Comparison of normalized DSIFs with different time interval $\Delta t$ : (a) the suddenly released bond model and (b) the transition bond model. 
(a)

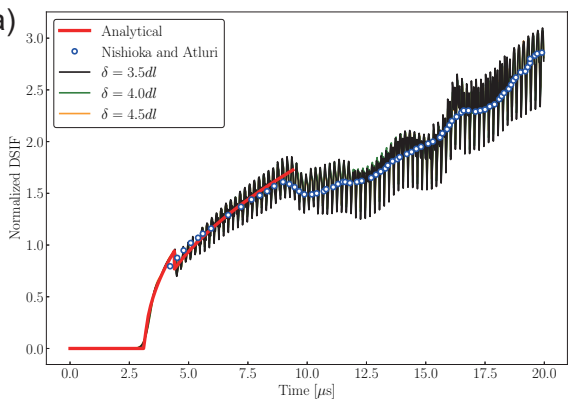

(b)

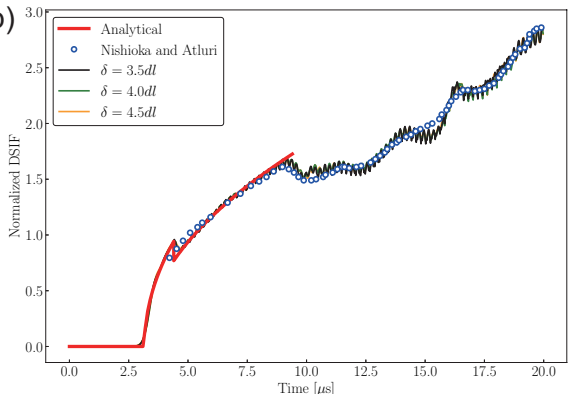

Figure 10: Comparison of normalized DSIFs with different horizon $\delta$ : (a) the suddenly released bond model and (b) the transition bond model.

(a)

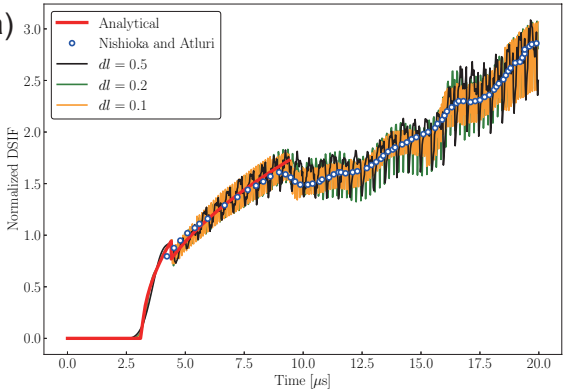

(b)

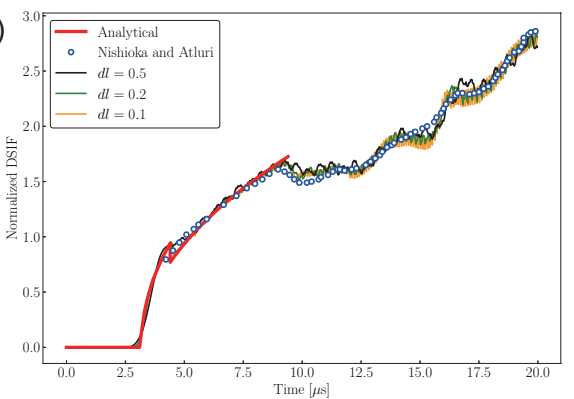

Figure 11: Comparison of normalized DSIFs with different particle distance $d l$ : (a) the suddenly released bond model and (b) the transition bond model. 


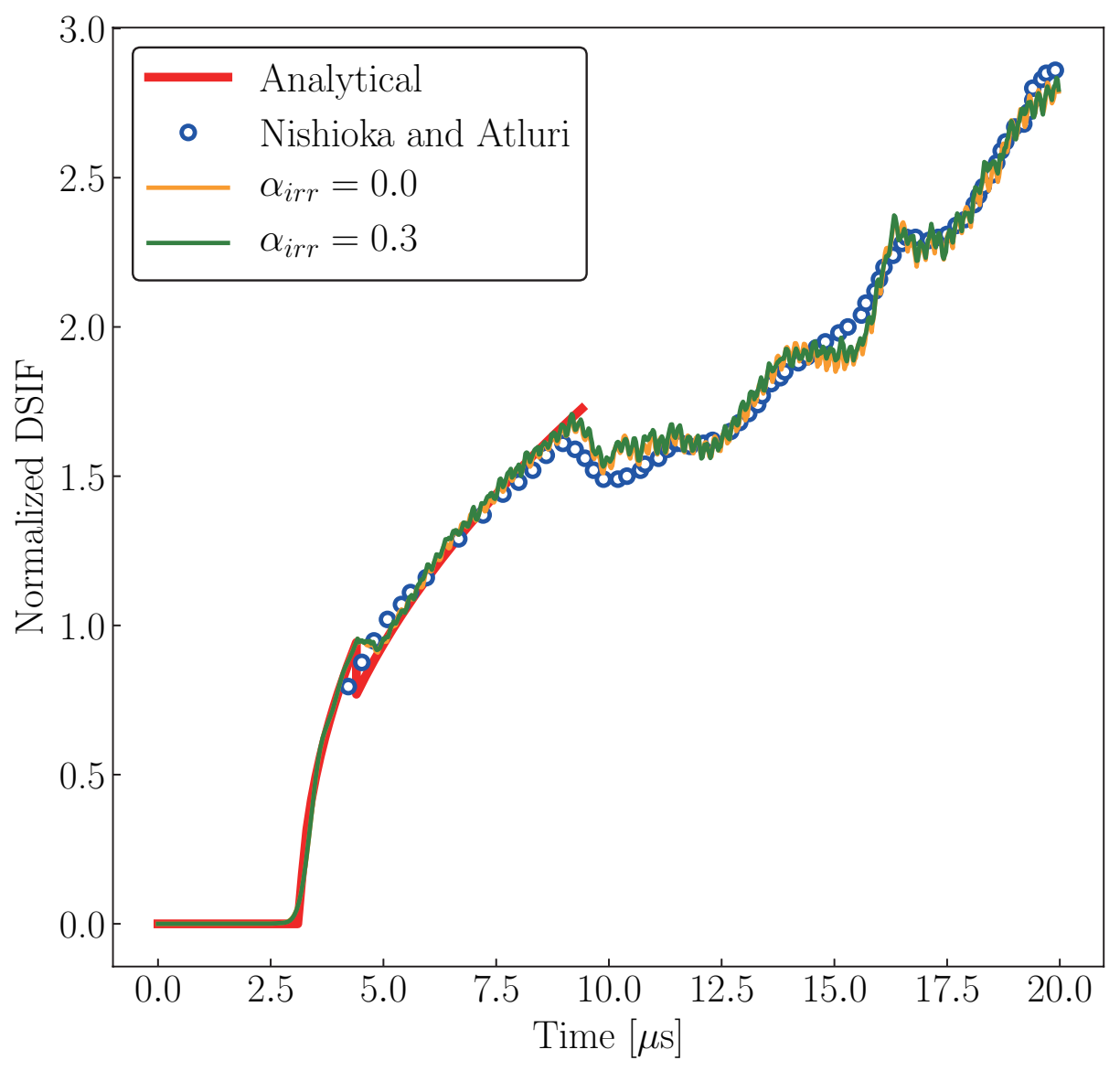

Figure 12: Comparison of normalized DSIFs with different particle arrangements. 
On the other hand, in the transition bond model, the reaction force does not oscillate. Therefore, the effectiveness of the transition bond is demonstrated for the load-displacement curve.

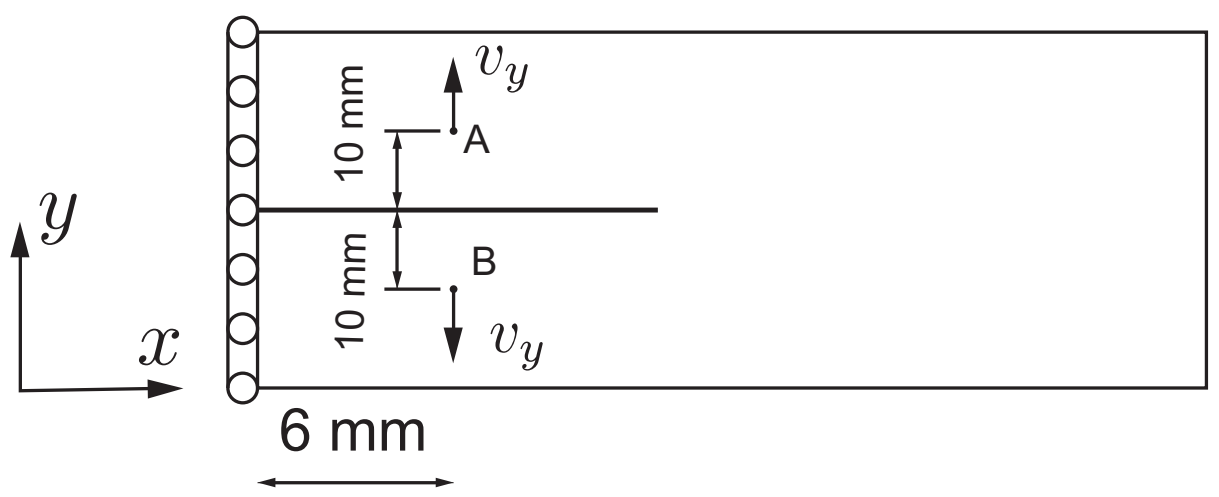

Figure 13: The cracked plate subjected to tensile loading.

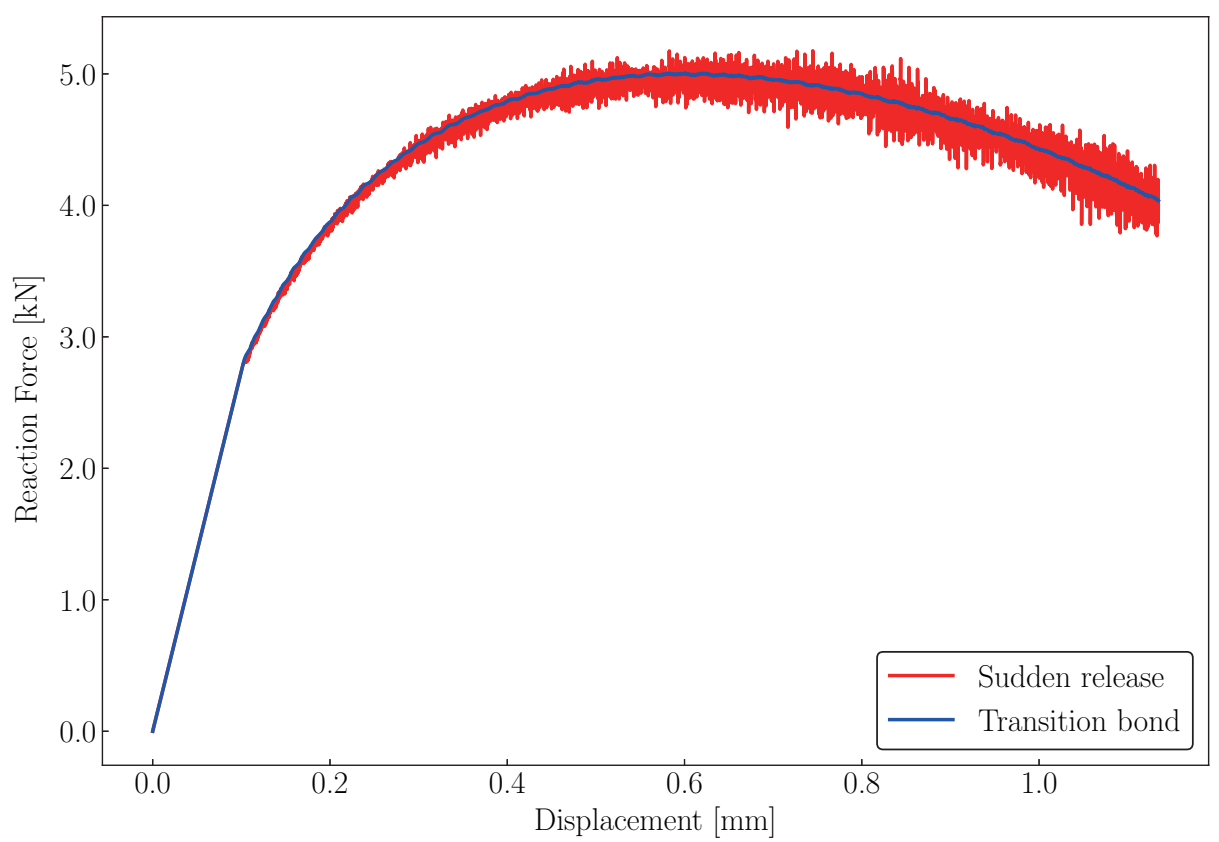

Figure 14: Load-deflection curve at point A. 


\subsection{Mixed-mode crack propagation problem}

The Kalthoff-Winkler impact test [32] is considered as a mixed-mode crack propagation problem. In the test, a projectile impacts the plate and a large shear load is applied to the crack tip and the crack propagates at an angle. In experiments, the crack angle is observed to be about $70^{\circ}$. A half model is employed because of the symmetry of the problem. The prescribed velocity is applied as the impact condition, and the analysis model is shown in Fig. 15. The height and width are $100 \mathrm{~mm}$ and the crack length is $50 \mathrm{~mm}$. The impact velocity is $16.5 \mathrm{~m} / \mathrm{s}$. The Young's modulus is $E=190.0 \mathrm{GPa}$, Poisson's ratio is $\nu=0.3$, the mass density is $\rho=8,000 \mathrm{~kg} / \mathrm{mm}^{3}$, and the fracture toughness is $K_{\text {Ic }}=68 \mathrm{MPa} \sqrt{\mathrm{m}}$. The crack velocity is $\dot{a}=750 \mathrm{~m} / \mathrm{s}$ [10]. The crack angle is evaluated by the maximum circumferential stress criterion. $r_{q 1}=2.0 \mathrm{~mm}$ and $r_{q 2}=4.0 \mathrm{~mm}$ are chosen for computing DSIFs. DSIFs of mode-I and -II are computed by Eq.(29). DSIFs are evaluated in regular and irregular particle arrangements. The irregularity factors $\alpha_{\text {irr }}=$ 0.3 and 0.6 are used. The next crack tip position is evaluated along the crack propagation direction $\theta_{c}$, as

$$
\begin{aligned}
& x=\Delta t \cdot \dot{a} \cos \left(\theta_{c}\right), \\
& y=\Delta t \cdot \dot{a} \sin \left(\theta_{c}\right) .
\end{aligned}
$$

DSIFs are evaluated at $\alpha_{\text {irr }}=0.0,0.3$, and 0.6, as shown in Fig.16(a). The DSIFs are in good agreement with each arrangement. The crack paths are shown in Fig.16(b), and the overall crack propagation angle is about $73.6^{\circ}$. The crack paths and crack angles are in good agreement with the experimental result. Fig.16(a) shows that the mode-I DSIF is positive and the mode-II DSIF is close to zero in accordance with the maximum circumferential stress theory. 


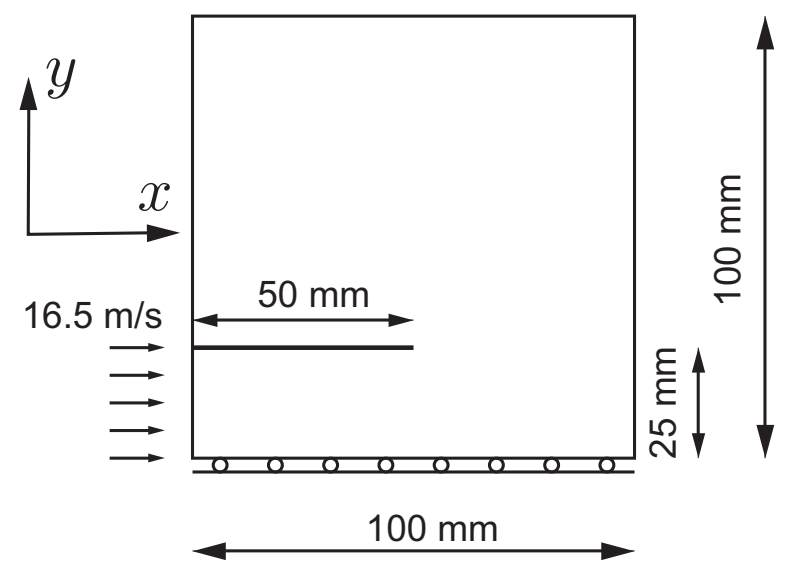

Figure 15: Analysis model of the Kalthoff-Winkler experiment. 
(a)

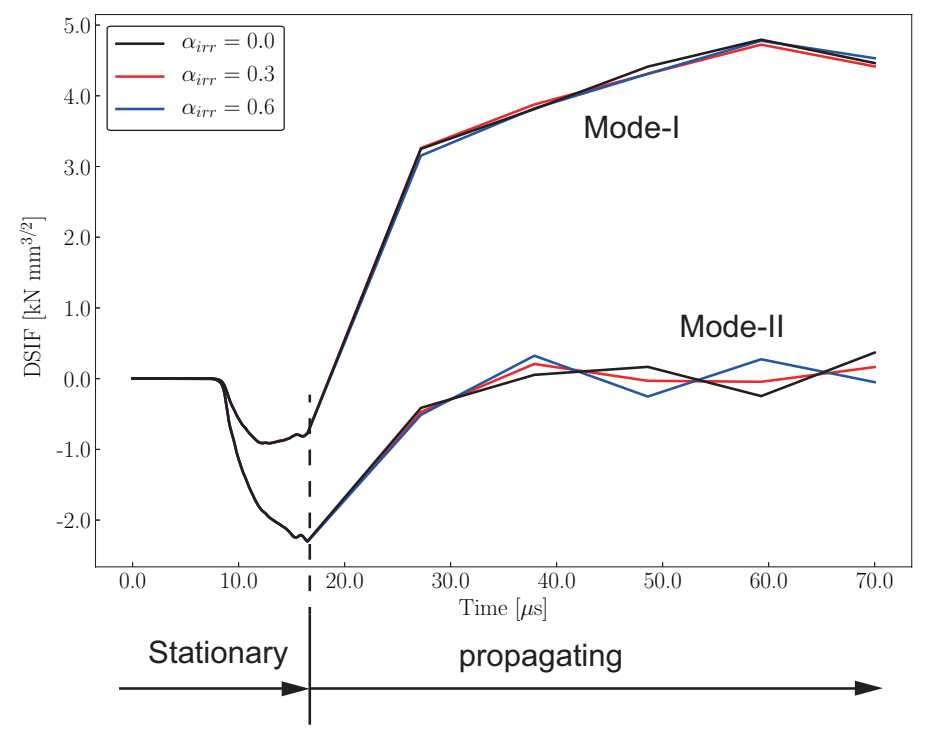

(b)

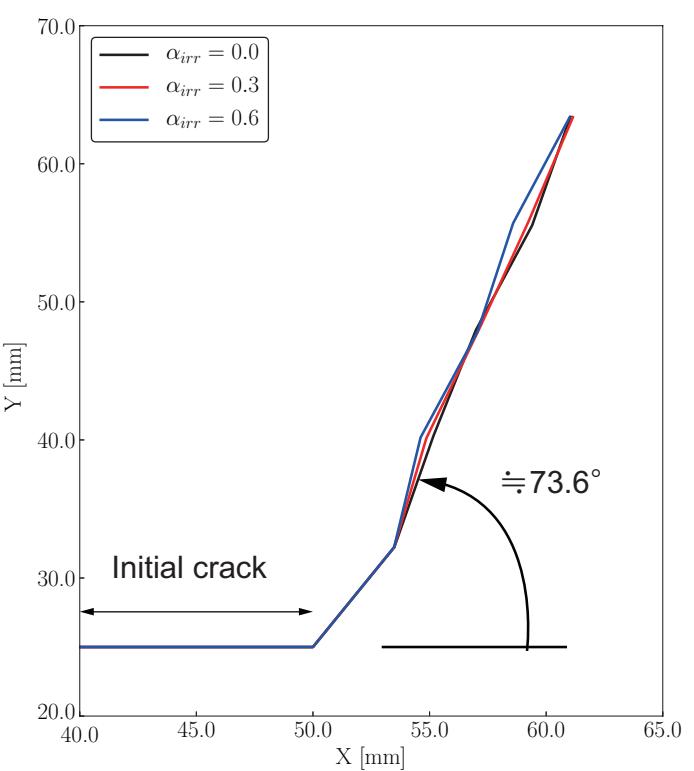

Figure 16: Numerical results with several particle arrangements: (a) DSIFs and (b) crack paths. 


\section{Conclusion}

The main objectives of the present work were to accurately evaluate the DSIFs and model dynamic crack propagation using PD theory by reducing numerical oscillation. Some major findings are summarized as follows. It was shown in the first numerical example that the numerical oscillations become significant for the suddenly released model and should be removed. The crack initiation criterion and the crack propagation direction cannot be evaluated well due to these oscillations. Additionally, the ratio of mode-I and mode-II DSIF is required when the direction is evaluated by the maximum circumferential stress criterion. Therefore, the oscillations are again important. To suppress the numerical oscillations, we proposed a new concept of the bond state, named as the transition bond. In the standard bond failure model, the connected bond becomes the disconnected bond when the crack is generated or grows. In the present concept, a connected bond first becomes a transition bond, then a disconnected bond when a crack crosses the line segment of a bond. The bond force decreases gradually in the transition bond. The effectiveness of the transition bond was examined using several damping lengths and damping coefficients. DSIFs for the irregular particle arrangement were also in good agreement with the reference solution. The Kalthoff-Winkler impact test was simulated as the mixed-mode crack propagation problem. The crack path of several particle arrangements agreed well with the experimental result. Furthermore, it is straightforward to extend the proposed technique to three dimensional problems because the transition effect is introduced when a bond is broken. Therefore, the proposed method is general and it is potentially applicable to other complex dynamic fracture, e.g., crack branching [33-35].

As a conclusion, the bond failure description based on sudden release model such as PMB model causes numerical oscillations when a bond is suddenly broken. The effect of the oscillations for different failure models should be further investigated which will be considered in a future study.

\section{Acknowledgements}

This research was partially supported by Sasakawa Scientific Research Grant from The Japan Science Society and the JSPS Grants-in-Aid for Scientific Research (C)(18K04582). 


\section{References}

[1] L.B. Freund, Dynamic fracture mechanics, Cambridge Monographs on Mechanics, 1990.

[2] K.Ravi-Chandar, Dynamic fracture, Elsevier, 2004.

[3] T.J.R. Hughes, The finite element method: linear static and dynamic finite element analysis, Dover Publication, 2000.

[4] N. Moës, J. Dolbow, T. Belytschko, A finite element method for crack growth without remeshing, Int. J. Numer. Meth. Eng. 46 (1999) 131150.

[5] T. Belytschko, Y.Y. Liu, L. Gu, Element-free Galerkin methods, Int. J. Numer. Meth. Eng. 37 (1994) 229-256.

[6] W.K. Liu, S. Jun, Y.F. Zhang, Reproducing kernel particle methods, Int. J. Numer. Meth. Fluid. 20 (1995) 1081-1106.

[7] S. Aoki, K.Kishimoto, H. Kondo, M. Sakata, Elastodynamic analysis of by finite element method using singular element Int. J. Fract. 14 (1978) 59-68.

[8] S. Aoki, K.Kishimoto, M. Sakata, Finite element computation of dynamic stress intensity factor for a rapidly propagation crack using $\hat{J}_{-}$ integral Comput. Mech. 2 (1987) 54-62.

[9] K. Kishimoto, S. Aoki, M. Sakata, Computer simulation of fast crack propagation in brittle material Int. J. Fract. 16 (1980) 3-13.

[10] J. Réthoré, A. Gravouil, A. Combescure, An energy-conserving scheme for dynamic crack growth using the extended finite element method, Int. J. Numer. Meth. Eng. 63 (2005) 631-659.

[11] T. Menouillard, J.Réthoré, A. Combescure, H. Bung, Efficient explicit time stepping for the extended finite element Method (X-FEM), Int. J. Numer. Meth. Eng. 68 (2006) 911-939.

[12] B. Prabel, A. Combescure, A. Gravouil, S. Marie, Level set X-FEM non-matching meshes: application to dynamic crack propagation in elastic-plastic media Int. J. Numer. Meth. Eng. 69 (2007) 1553-1569. 
[13] D. Greégoire, H Maigre, J.Réthoré, A. Combescure, Dynamic crack propagation under mixed-mode loading - Comparison between experiments and X-FEM simulations Int. J. Solid. Struct. 44 (2007) 65176534 .

[14] S.A. Silling, Reformulation of elasticity theory for discontinuities and long-range forces, J. Mech. Phys. Solid. 48 (2000) 175-209.

[15] S.A. Silling, E. Askari, A meshfree method based on the peridynamic model of solid mechanics, Comput. Struct. 83 (2005) 1526-1535.

[16] S.A. Silling, M. Epton, O. Weckner, J. Xu, E. Askari, Peridynamic states and constitutive modeling, J. Elasticity. 88 (2007) 151-184.

[17] H. Ren, X. Zhuang, Y. Cai, T. Rabczuk, Dual-horizon peridynamics, Int. J. Numer. Meth. Eng. 108 (2016) 1451-1476.

[18] H. Ren, X. Zhuang, T. Rabczuk, Dual-horizon peridynamics: A stable solution to varying horizons, Comput. Method Appl. Mech. Engrg. 318 (2017) 762-782.

[19] B. Kilic, E. Madenci, Prediction of crack paths in a quenched glass plate by using peridynamic theory, Int. J. Fract. 156 (2009) 165-177.

[20] Y.D. Ha, F. Bobaru, Studies of dynamic crack propagation and crack branching with peridynamics, Int. J. Fract. 162 (2010) 229-244.

[21] Y.D. Ha, F. Bobaru, Characteristics of dynamic brittle fracture captured with peridynamics, Eng. Fract. Mech. 78 (2011) 1156-1168.

[22] A. Agwai, I. Guven, E. Madenci, Predicting crack propagation with peridynamics: a comparative study, Int. J. Fract. 171 (2011) 65-78.

[23] M. Imachi, S. Tanaka, Dynamics stress intensity factors evaluation employing ordinary state-based peridynamics, Trans. JSCES, 2016 (2016) 20160017. (in Japanese)

[24] M. Imachi, S. Tanaka, T.Q. Bui, Mixed-mode dynamic stress intensity factors evaluation using ordinary state-based peridynamics Theor. Appl. Fract. Mech. 93 (2018) 97-104. 
[25] Q.V. Le, W.K. Chan, J. Schwartz, A two-dimensional ordinary, statebased peridynamic model for linearly elastic solids, Int. J. Numer. Meth. Eng. 98 (2014) 547-561.

[26] P. Lancaster, K. Salkauskas, Surfaces generated by moving least squares methods, Math. Comput. 37 (1981) 141-158.

[27] D. Organ, M. Fleming, T. Terry, T. Belytschko, Continuous meshless approximations for nonconvex bodies by diffraction and transparency, Comput. Mech. 18 (1996) 225-235.

[28] W. Hu, Y.D. Ha, F. Bobaru, S.A. Silling, The formulation and computation of the nonlocal J-integral in bond-based peridynamics, Int. J. Fract. 176 (2012) 195-206.

[29] R. Panchadhara, P.A. Gordon, Application of peridynamic stress intensity factors to dynamic fracture initiation and propagation, Int. J. Fract. 201 (2016) 81-96.

[30] F. Erdogan, G. C. Sih, On the crack extension in plates under plane loading and transverse shear, J. Basic. Eng. 85 (1963) 519-527.

[31] T. Nishioka, S.N. Atluri, Numerical modeling of dynamic crack propagation in finite bodies, by moving singular elements, J. Appl. Mech. 47 (1980) 577-582.

[32] J. F. Kalthoff, S. Winkler, Failure mode transition at high rates of shear loading, Impact loading and dynamic behavior of materials, (1987) 185195.

[33] T. Rabczuk, T. Belytschko, Cracking particles: a simplified meshfree method for arbitrary evalving cracks, Int. J. Numer. Meth. Eng. 61 (2004) 2316-2343.

[34] T. Rabczuk, T. Belytschko, A three-dimensional large deformation meshfree method for arbitray evolving cracks Comput. Method Appl. Mech. Engrg. 196 (2007) 2777-2799.

[35] T. Rabczuk, G. Zi, S. Bordas, H. Nguyen-Xuan, A simple and robust three-dimensional cracking-particle method without enrichment Comput. Method Appl. Mech. Engrg. 199 (2010) 2437-2455. 
Nomenclature

Latin symbols

$a, \dot{a}$ : crack length and crack velocity

$A_{I}, A_{I I}$ : universal function for Eq.(5)

$\boldsymbol{b}$ : external force vector

$c_{d}, c_{s}, c_{\mathrm{R}}:$ longitudinal, transvers Rayleigh wave speed

$d a$ : crack growth length

$d l$ : particle distance

$D$ : parameter for universal function $A_{I}, A_{I I}$

$E, E^{*}$ : Young's modulus and modified Young's modulus for plane stress/strain condition

$f_{b}$ : damping function

$I$ : interaction integral

$k(v)$ : coefficient depends on velocity $v$ in Eq.(13)

$K_{i}^{\text {act }}, K_{i}^{\text {aux }}(i=\mathrm{I}, \mathrm{II}):$ SIF of actual and auxiliary field

$K_{\theta_{c}}$ : equivalent SIF

$K_{\text {Ic }}:$ fracture toughness

$\bar{K}:$ normalized SIF

$m$ : unit direction vector

$n$ : damping coefficient

$q_{i}, q_{i, j}$ : weight function and its derivative for interaction integral

$r_{q}, r_{q 1}, r_{q 2}:$ parameters of interaction integral

$r_{c}$ : randomization factor

$\underline{t}$ : magnitude for force density

$t_{c r}:$ critical time for crack growth

$T$ : force density vector

$\boldsymbol{u}, \dot{\boldsymbol{u}}, \ddot{\boldsymbol{u}}$ : displacement, velocity and acceleration vectors

$u_{i}^{\text {act }}, u_{i}^{\text {aux }}:$ components of displacement on actual and auxiliary field in Eq.(3)

$u_{i, j}^{\text {act }}, u_{i, j}^{\text {aux }}:$ derivatives of $u_{i}^{\text {act }}, u_{i}^{\text {aux }}$ with respect to $x_{j}$ in Eq.(3)

$\dot{u}_{i}^{\text {act }}, \dot{u}_{i}^{\text {aux }}$ : components of velocity of actual and auxiliary field in Eq.(3)

$\dot{u}_{i, j}^{\text {act }}, \dot{u}_{i, j}^{\text {aux }}:$ derivatives of $\dot{u}_{i}^{\text {act }}, \dot{u}_{i}^{\text {aux }}$ with respect to $x_{j}$ in Eq.(3)

$V$ : volume

$\boldsymbol{x}:$ position vector 


\section{Greek symbols}

$\alpha_{d}, \alpha_{s}:$ parameter for universal function $A_{I}, A_{I I}$

$\alpha_{i r r}:$ irregularity factor

$\delta$ : horizon

$\Delta a$ : damping length

$\boldsymbol{\eta}$ : relative displacement vector

$\theta_{c}:$ crack propagation direction

$\kappa$ : parameter for universal function $A_{I}, A_{I I}$

$v$ : Poisson's ratio

$\xi$ : relative position vector

$\rho$ : mass density

$\sigma_{0}:$ applied stress

$\sigma_{i j, k}^{\text {act }}, \sigma_{i j, k}^{\text {aux }}:$ derivative of $\sigma_{i j}^{\text {act }}, \sigma_{i j}^{\text {aux }}$ with respect to $x_{k}$

$\sigma_{i j}^{\text {act }}, \sigma_{i j}^{\text {aux }}:$ component of stress on actual and auxiliary field

\section{Abbreviations}

PD : peridynamics

OSPD : ordinary state-based peridynamics

SIF : stress intensity factor

DSIF : dynamic stress intensity factor

FEM : finite element method

$\mathrm{X}$-FEM : extended finite element method

PMB : prototype microelastic brittle

MLSA : moving least squares approximation 